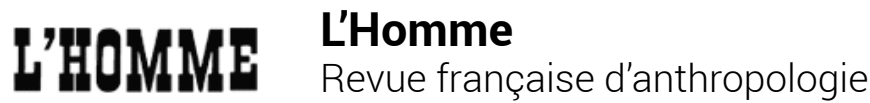

239-240 | 2021

Expériences initiatiques du genre

\section{Andrew Beatty, Emotional Worlds. Beyond an Anthropology of Emotion}

\section{Olivier Allard}

\section{(2) OpenEdition}

1 Journals

\section{Édition électronique}

URL : https://journals.openedition.org/lhomme/41319

DOI : 10.4000//homme.41319

ISSN : 1953-8103

Éditeur

Éditions de l'EHESS

\section{Édition imprimée}

Date de publication : 1 décembre 2021

Pagination : 333-336

ISBN : 978-2-7132-2868-1

ISSN : 0439-4216

\section{Référence électronique}

Olivier Allard, « Andrew Beatty, Emotional Worlds. Beyond an Anthropology of Emotion », L'Homme [En ligne], 239-240 | 2021, mis en ligne le 01 décembre 2021, consulté le 15 décembre 2021. URL : http:// journals.openedition.org//homme/41319; DOI : https://doi.org/10.4000//homme.41319

Ce document a été généré automatiquement le 15 décembre 2021.

(c) École des hautes études en sciences sociales 


\title{
Andrew Beatty, Emotional Worlds. Beyond an Anthropology of Emotion
}

\author{
Olivier Allard
}

\section{RÉFÉRENCE}

Andrew Beatty, Emotional Worlds. Beyond an Anthropology of Emotion. Cambridge, Cambridge University Press, 2019, 305 p., notes bibliogr., bibl., index (« New Departures in Anthropology »).

1 L'anthropologie des émotions est un courant qui a connu son heure de gloire dans le dernier tiers du xxe siècle, mais semble aujourd'hui délaissé, en partie concurrencé par d'autres approches, telles que celles participant du tournant affectif. Andrew Beatty nous offre un ouvrage qui a l'immense mérite de revenir sur cette importante tradition tout en proposant bien plus qu'une simple synthèse : il y approfondit aussi sa propre idée de ce que doit être une «anthropologie émotionnellement impliquée [emotionally engaged anthropology]» (p. 12).

2 Andrew Beatty rappelle avec clarté les débats qui ont animé l'anthropologie des émotions, présente en détail les grandes monographies qui ont marqué le champ et montre les échanges possibles avec les recherches menées en philosophie et en psychologie. Il revient également sur les précautions méthodologiques qu'il avait développées dans des publications précédentes ${ }^{1}$, en soulignant la difficulté à reconnaître et à interpréter les épisodes émotionnels sur le terrain. Plutôt que d'évacuer ces questionnements en un chapitre préliminaire, Beatty met en garde son lectorat tout au long du livre contre les raccourcis trop faciles. Les termes émotionnels ne renvoient pas nécessairement à des expériences vécues, mais peuvent aussi relever de la rhétorique politique, comme l'auteur a pu l'observer sur la petite île indonésienne de Nias (p. 62). Dans d'autres contextes, comme à Java, le ressenti peut inversement jouer un rôle crucial pour guider les interactions, en permettant d'évaluer la situation et sa propre position : les émotions sont une manière de sentir le terrain (« feeling your 
way ", en anglais, est une formulation sans doute encore plus parlante, p. 83). Avoir mené successivement deux véritables terrains à Nias et à Java représente donc pour Beatty un immense atout. En s'appuyant sur le contraste entre ces deux sociétés insulaires, il montre que les émotions ne constituent pas un domaine qui fonctionnerait partout de la même manière: il est faux d'affirmer que les émotions ne sont que rhétoriques, ou que les termes vernaculaires désignent toujours des réalités vécues. Ce ne sont que des possibilités parmi d'autres. Pour trancher la question, Beatty ne propose pas de solution particulière : il fait confiance à la pratique ethnographique, à notre capacité à saisir ce que disent, font, ou vivent les personnes avec lesquelles nous cohabitons durablement sur le terrain.

3 Tout en refusant de figer une définition des émotions comme point de départ ou comme objectif de l'enquête, Andrew Beatty en adopte une acception assez stricte, inspirée notamment par la philosophie et la psychologie d'orientation cognitive : les émotions sont des événements transitoires, des épisodes circonscrits, qui se démarquent donc de l'humeur (mood) ou de l'ethos. On peut les distinguer par différents scénarios typiques et, de ce point de vue, les émotions sont essentiellement des événements temporels, des histoires plus que des états ou des représentations (p. 116). L'originalité de Beatty consiste alors à souligner qu'on ne peut comprendre ces épisodes momentanés qu'en les replaçant dans l'histoire longue des personnes impliquées et de leurs relations: c'est la dimension intrinsèquement narrative des émotions. Si les émotions sont souvent liées à des objectifs et à des désirs individuels satisfaits ou frustrés (p.160), donc à un certain rapport au futur, Beatty met en évidence le fait qu'elles s'enracinent dans le passé, qu'elles représentent même «le passé dans le présent » (p. 176).

4 C'est l'un des principaux enjeux de l'écriture ethnographique : il faut à la fois faire le récit détaillé d'épisodes émotionnels - telle scène d'humiliation ou crise de colère - et celui des relations dans lesquelles ils s'inscrivent. En effet, si les émotions sont par nature «ce qui m'affecte personnellement comme individu " (p. 20), des « expériences à la première personne » (p. 126), alors il est impossible de les comprendre en restant au niveau de scénarios abstraits ou de considérations générales, comme Andrew Beatty reproche à Michelle Rosaldo ou Catherine Lutz de le faire. En outre, le contexte immédiat est généralement beaucoup trop mince pour nous permettre de saisir des événements qui plongent leurs racines dans le passé. C'est la raison pour laquelle il se montre très critique à l'égard des travaux de Clifford Geertz, ou même de Robert Levy. Il n'y a guère que Jean Briggs et son fameux Never in Anger $(1970)^{2}$ qui trouvent grâce à ses yeux. L'insuffisance ethnographique représente en fait le principal grief de Beatty envers ses prédécesseurs: les émotions des autres paraissent souvent mystérieuses, tout simplement parce qu'on n'en sait pas assez (p. 185). Il défend donc le principe d'un « engagement avec les autres, pas avec les Autres » (p. 262), c'est-à-dire la nécessité de donner la priorité au tissu des relations interpersonnelles par rapport à une altérité culturelle parfois trompeuse. Plus que l'explication de grands concepts, il nous manque, la plupart du temps, l'histoire, le contexte, les personnages, l'intrigue (p. 183). Andrew Beatty tire de son propre travail des drames parfois noués dès la jeunesse des protagonistes, voire à la génération précédente (p.190), qui sont comme des échantillons de l'anthropologie qu'il défend, même s'il reconnaît ne pas pouvoir les détailler suffisamment. 
5 Si cette insistance sur l'ethnographie ne peut être que louable, elle recouvre cependant deux éléments plus ambivalents. D'une part, Andrew Beatty problématise beaucoup plus l'écriture que le terrain. Même lorsqu'il montre une connaissance fine des milieux qu'il a étudiés, il apparaît singulièrement détaché au point de revendiquer explicitement une stricte séparation entre les préoccupations personnelles de l'ethnographe et celles des populations locales (p.152). Il balaie ici peut-être un peu rapidement toutes les réflexions sur la possibilité d'être affecté sur le terrain, expérience qui est pourtant, pour beaucoup d'anthropologues, indispensable. D'autre part, Beatty a recours, à de nombreuses reprises, à la littérature, comme modèle pour l'ethnographie ou pour y puiser des exemples, en soulignant notamment qu'elle permet de contourner le problème de l'accès à l'intériorité d'autrui: contrairement à l'ethnographe, le romancier peut être omniscient (pp. 25, 150). Un tel présupposé me semble cependant totalement erroné, car il s'agit alors, au mieux, de la manière dont cette intériorité est imaginée, mais certainement pas du ressenti lui-même. Notons par ailleurs que Beatty ne se réfère pas à n'importe quelle littérature; il cite principalement les grands auteurs du XIXe et du début du $\mathrm{xx}^{\mathrm{e}}$ siècle (Tolstoï, Forster, Conrad ou encore Musil). Ce choix montre bien qu'il s'appuie en réalité sur une conception assez particulière et située du sujet, dont l'individualité est nourrie par les relations familiales et affectives, et dont le comportement est fondamentalement motivé par l'intériorité. On comprend ainsi sa critique des approches en termes d'affect, généralement rassemblées sous l'expression d'affect theory : c'est selon lui de la mauvaise science, mais aussi de la mauvaise littérature. Les études de ce courant relèvent souvent d'exercices stylistiques jargonnants, où l'action se situe sur la page plus que sur le terrain (p. 211). L'ethnographie est alors escamotée par l'omniprésence des références théoriques; surtout, elle prend une forme qui ne satisfait guère Beatty. En effet, à vouloir s'intéresser à l'affect comme énergie qui circule entre les personnes et les choses, par opposition aux émotions qui représenteraient avant tout des catégories sémantiques et culturelles, les anthropologues de ce courant n'accordent finalement qu'une importance secondaire à la parole de leurs interlocuteurs et interlocutrices. Yael Navaro-Yashin, par exemple, a tenté de dépeindre directement la charge affective de documents administratifs ou de ruines sur les Chypriotes turcs ${ }^{3}$, en allant parfois même à l'encontre des témoignages qu'elle avait recueillis sur place - ce qui ne laisse pas de choquer Beatty (p. 224). Pour ce dernier, le sujet humain doit, par définition, rester au centre de l'anthropologie ; les objets n'ont une charge affective que dans la mesure où des personnes en font l'expérience, ou bien encore parce qu'elles leur attribuent une telle signification. Nombre d'anthropologues seront sans doute d'accord avec ce rappel de bon sens, mais le tournant affectif a aussi d'autres objectifs qui ne sont pas dénués de pertinence. Parler d'affect (au singulier) vise en effet aussi à réintroduire de l'indétermination dans le tableau trop léché des diverses émotions qu'on les différencie par leurs noms ou par leurs scénarios typiques. Or, c'est bien là un but qui semble totalement étranger aux préoccupations de Beatty. S'il prend soin de discuter le rapport complexe entre les termes émotionnels et les phénomènes que ces derniers désignent (chap. IX), savoir distinguer les diverses émotions constitue selon lui un préalable à toute recherche (exemple pp. 64,110 ). Faut-il pour autant camper sur cette position?

6 Dans son panorama très complet, Andrew Beatty oublie un courant de recherches qui lui aurait peut-être permis de prendre au sérieux la critique des émotions comme catégories délimitées, sans renoncer à ses exigences de rigueur descriptive: 
l'anthropologie linguistique. À sa décharge, il faut reconnaitre que les études exceptionnelles menées dans ce cadre, dans les années 1980-1990, sont très peu présentes dans les débats actuels autour des émotions et de l'affect. Les principaux représentants de l'anthropologie linguistique qui se sont intéressés au sujet - tels Niko Besnier, Judith Irvine, Elinor Ochs ou Bambi Schieffelin - n'ont en effet jamais mis au cœur de leurs recherches la discussion des termes qui désignent les différentes émotions. C'est au contraire la présence de l'affect dans toutes les autres dimensions du langage qui a retenu leur attention, du choix de certaines métaphores à celui des évidentiels, en passant par les idéophones, la hauteur de voix ou le débit de parole. Beatty entrevoit, certes, cette dimension à plusieurs reprises, par exemple lorsqu'il relève l'effet émotionnel des pronoms, comme un «nous » inclusif plutôt qu'exclusif (p. 62), ou lorsqu'il remarque que les émotions peuvent être présentes sans être toutefois nommées (pp. 166, 233). Mais il met toujours au premier plan la discussion des termes émotionnels et leur signification référentielle, alors que de nombreux travaux ont souligné que les manières les plus explicites d'encoder l'affect dans le langage étaient les moins efficaces, qu'il était par exemple plus touchant d'exhiber sa peine (par des pleurs, une voix cassée ou des inspirations sonores) que de simplement dire qu'on est triste. Ces recherches ont aussi montré que l'indétermination de l'affect n'est pas un simple défaut de précision descriptive ou analytique - comme Beatty le reproche aux tenants de l'affect theory -, mais une conséquence de la nature multifonctionnelle des outils qui inscrivent l'affect dans le langage. Ils ne prennent tout leur sens que dans un contexte particulier et leur indétermination peut représenter une ressource pour les locuteurs (pensons, par exemple, aux différentes significations que peut recouvrir le tutoiement). Il est vrai que l'anthropologie linguistique pragmatique a généralement privilégié l'étude de moments circonscrits, de scènes se produisant dans des contextes d'interlocution précis et susceptibles d'être retranscrites intégralement, loin de la profondeur biographique que défend Beatty. C'est peut-être une différence d'orientation qui explique son peu d'intérêt, mais il est regrettable qu'il ait presque totalement ignoré ce courant, qui l'aurait sans doute conduit à nuancer considérablement certaines de ses thèses.

En définitive, cet ouvrage, qui reste d'une grande richesse, montre bien que les émotions ne doivent pas être cantonnées à un sous-champ de l'anthropologie et qu'elles possèdent donc un intérêt anthropologique général. En restant campé sur une position classique, Andrew Beatty ne parvient toutefois peut-être pas - en dépit de ce qui est annoncé dans le sous-titre - à vraiment aller « au-delà d'une anthropologie des émotions ».

\section{NOTES}

1. Cf. notamment Andrew Beatty, «Emotions in the Field: What Are We Talking About? ", Journal of the Royal Anthropological Institute, 2005, 11 (1) : 17-37. 
2. Jean Briggs, Never in Anger. Portrait of an Eskimo Family, Cambridge, Cambridge University Press, 1970.

3. Cf. Yael Navaro-Yashin, The Make-Believe Space. Affective Geography in a Postwar Polity, Durham, Duke University Press, 2012. 